\title{
UM MODELO DE MINIMIZAÇÃO DE CUSTOS EM DIAGNÓSTICOS COM UM CASO DE APLICAÇÃO EM UM BANCO DE SANGUE
}

Eugenio K. Epprecht

PUC-Rio / Departamento de Engenharia Industrial

R. Marquês de São Vicente, 225

Rio de Janeiro - RJ - 22453-900

e-mail: eke@rdc.puc-rio.br

Gutemberg Hespanha Brasil

UFES / Departamento de Estatística

Av. Fernando Ferrari s/ $\mathrm{n}^{\circ}$

Vitória - ES - 29060-900

e-mail: ghbrasil@zaz.com.br

Mário Cezar dos Santos Jr.

PUC-Rio / Departamento de Engenharia Industrial

R. Marquês de São Vicente, 225

Rio de Janeiro - RJ - 22453-900

\section{Resumo}

O problema aqui tratado é, dado um conjunto de testes para determinação de um diagnóstico, determinar a seqüência de execução destes testes com custo esperado mínimo. Assumindo algumas hipóteses simplificadoras, apresenta-se uma solução que fornece diretamente a seqüência ótima, eliminando a necessidade de busca. A solução se estende também a uma versão do problema com uma estrutura hierárquica de testes. O modelo é genérico, podendo aplicar-se a diagnósticos nos mais diversos contextos: de problemas de processos em controle de qualidade, de falhas de equipamentos, ou no contexto médico. É apresentada uma extensão do modelo, para situações em que algumas das hipóteses básicas do modelo original não se aplicam. Esta extensão foi motivada por um problema real, de minimização de gastos com exames sorológicos em um banco de sangue. A solução obtida para esse problema resultou em economia substancial.

Palavras-chave: diagnóstico, custo mínimo, aplicações à saúde.

\begin{abstract}
This work deals with the following problem: given a set of tests for a diagnosis problem, find the minimum-cost order of execution of the tests. It is shown that under some simplifying assumptions, graph search is obviated by a very straightforward solution. This solution is also applicable to situations in which the tests follow a hierarchical organization. The model is generic, with no restriction of context: it is applicable to the diagnosis of equipment failures, of problems with processes in the context of quality control, or to medical diagnosis. An extension of the basic model for the situation in which some of the simplifying assumptions are not applicable is presented. This extension was motivated by a real problem, and the solution obtained led to significant cost reduction.
\end{abstract}

Keywords: diagnosis, minimal cost, health applications. 


\section{Introdução}

A motivação inicial para a análise do problema central aqui tratado surgiu da constatação de que a literatura tradicional em Controle de Qualidade, que indica o "brainstorming" e o diagrama "espinha-de-peixe" como ferramentas para auxiliar no levantamento e enumeração do conjunto de causas possíveis para um problema observado, não indica nenhum procedimento para a identificação da causa responsável dentro deste conjunto (ver por exemplo Banks, 1989, e Montgomery, 1997). Evidentemente, os procedimentos específicos para identificação da(s) causa(s) - em uma palavra, para o diagnóstico - dependem da natureza e características particulares de cada processo ou equipamento, e ainda do ambiente em que ele se insere, o que explica facilmente a omissão.

Há, contudo, um problema ligado ao diagnóstico, que independe do contexto particular do processo ou equipamento em questão, e que pode surgir em muitas situações: dado um conjunto de testes possíveis para determinação da causa, cada um com um custo associado, em que ordem executar os testes de maneira a minimizar o custo total esperado do diagnóstico? Este é o problema que aqui será abordado.

Este problema não se restringe ao contexto de diagnóstico de falhas em equipamentos, ou de causas de problemas em processos. Por exemplo, o modelo e método de solução desenvolvidos foram aplicados a um problema concreto de diagnóstico em um banco de sangue, resultando em economia substancial.

Há trabalhos relacionados. Sem pretendermos ser exaustivos, no Brasil podem-se citar Goulart et al. (1994) e Pereira (1994). Os primeiros, porém, não consideram o fator custo que, no contexto industrial, bem como no contexto do caso analisado, do banco de sangue, é de importância fundamental. (No contexto de seu trabalho - de que são possíveis exemplos de aplicação: questionários, onde se quer minimizar o número de perguntas, e sistemas especialistas, onde se quer minimizar o tempo de processamento - o critério relevante é a minimização do número de testes - o que, em termos de modelo matemático, equivale a não considerar os custos dos testes, ou a considerar implicitamente que todos os testes possuem custo igual). Pereira (1994) considera o custo explicitamente, mas trata o problema no contexto bem mais específico de uma classe de problemas de diagnóstico de falhas de equipamento, e no nível de modelagem das relações particulares de causa e efeito para o diagnóstico do tipo de equipamento em questão. Neste nível mais específico, desenvolve um modelo baseado em heurísticas para solução por sistemas a base de conhecimentos. No contexto do presente trabalho, o interesse é por um modelo genérico e que considere os custos dos testes; nisto ele se distingue dos trabalhos citados.

As próximas seções apresentam: inicialmente (Seção 2.1) a formulação matemática para o problema, incluindo algumas hipóteses simplificadoras; estas, embora demasiado restritivas em relação à maioria das situações reais típicas, permitem uma solução elegante para o modelo (Seção 2.2), que serve de base para a análise de situações mais complexas (uma dessas situações é mencionada brevemente na Seção 2.3). O modelo básico é então estendido (3.1), para o caso em que uma das hipóteses simplificadoras é violada, sendo desenvolvido um algoritmo para solução (3.2). É descrito, em seguida, o problema concreto do banco de sangue (4.1), e discutidos os resultados de sua solução pelo algoritmo: economia proporcionada, questões de implementação da solução na prática, e análise de sensibilidade (seções 4.2 a 4.4). Nas conclusões e comentários finais (Seção 5) discutem-se o ganho proporcionado pelo algoritmo em termos de redução do espaço de busca, o leque de aplicações potenciais do modelo, e direções para pesquisa futura. 


\section{Modelo para o Caso de Diagnósticos Mutuamente Exclusivos}

\subsection{Formulação do problema}

Dado o conjunto $\mathrm{D}=\left\{\mathrm{d}_{1}, \mathrm{~d}_{2}, \ldots \mathrm{d}_{\mathrm{n}}\right\}$ das $n$ causas possíveis de uma falha ou problema - ou, mais genericamente, um conjunto de diagnósticos possíveis - deseja-se determinar a causa responsável - ou o diagnóstico correto. (Daqui por diante, referir-se-á sempre a “diagnósticos”). São feitas as seguintes hipóteses simplificadoras: (a) os diagnósticos em D são mutuamente exclusivos e exaustivos: um e somente um é o correto; (b) para cada diagnóstico $\mathrm{d}_{\mathrm{i}} \in \mathrm{D}$, existe um e apenas um teste $\mathrm{t}_{\mathrm{i}}$ que permite determiná-lo; (c) o resultado do teste indica com $100 \%$ de certeza um dos dois eventos seguintes: o diagnóstico correto é $\mathrm{d}_{\mathrm{i}}$; ou o diagnóstico correto não é $\mathrm{d}_{\mathrm{i}}$.

Cada diagnóstico $\mathrm{d}_{\mathrm{i}}$ possui uma probabilidade a priori $\mathrm{p}_{\mathrm{i}}$ de ser o correto (estimada a partir de estatísticas passadas, ou de conhecimento do processo, ou ainda subjetivamente). Na execução de cada teste $t_{i}$ incorre-se em um custo $c_{i}$ (este custo engloba tanto os custos diretos de execução do teste, como quaisquer custos indiretos em que a execução do teste implique, tais como custos de interrupção da produção ou de demora na correção do problema). Assim, há um conjunto de $n$ testes, T, um conjunto de $n$ probabilidades, $\mathrm{P}$, e um conjunto de $n$ custos, C, de tal forma que há uma associação entre os elementos de mesmo índice nos conjuntos D, T, P e C. Além disso, $\Sigma \mathrm{p}_{\mathrm{i}}=1$.

O procedimento de investigação da causa responsável (determinação do diagnóstico) começa pela escolha de um primeiro teste $t_{i 1}$ a ser executado. Se o resultado for positivo, a causa foi identificada (o diagnóstico determinado) e o processo termina; se negativo, um segundo teste $t_{\mathrm{i} 2}$ deve ser escolhido e realizado, e assim por diante. ( $\mathrm{O}$ duplo índice é usado para distinguir entre os índices de posição dos testes na seqüência de execução e os seus "índices-nome" dentro do conjunto T; desta forma $i j$ representa o índice-nome do teste que ocupa a $j$-ésima posição na seqüência de execução. Por exemplo, na seqüência $\left(t_{3}, t_{5}, t_{1}, t_{2}, t_{4}\right)$, tem-se $i_{1}=3$, $i_{2}=5, i_{3}=1, i_{4}=2$ e $\left.i_{5}=4\right)$.

Como a seqüência é interrompida assim que a causa for identificada (assim que se chegar a um diagnóstico) — não se sabendo a priori quando isto ocorrerá — o custo da seqüência de testes efetivamente realizados é uma variável aleatória. A função-objetivo a minimizar é o valor esperado deste custo. Este valor esperado é função, obviamente, dos valores das probabilidades em $\mathrm{P}$ e dos custos em $\mathrm{C}$ (que constituem os dados do problema), bem como da ordem de execução dos testes. A decisão é em relação à ordem de execução dos testes: a variável de decisão é o vetor, ou seqüência, de índices $\left(\mathrm{i}_{1}, \mathrm{i}_{2}, \ldots, \mathrm{i}_{\mathrm{n}}\right)$, que indica esta ordem de execução. Daqui em diante, utilizar-se-á o termo "seqüência" para designar o conjunto dos testes executados segundo uma certa ordem.

É fácil verificar que, para uma dada ordem de execução dos testes, o valor esperado do custo total $\mathrm{C}_{\mathrm{T}}$ é dado pela seguinte expressão:

$$
\mathrm{E}\left(\mathrm{C}_{\mathrm{T}}\right)=\mathrm{p}_{\mathrm{i} 1} \mathrm{c}_{\mathrm{i} 1}+\mathrm{p}_{\mathrm{i} 2}\left(\mathrm{c}_{\mathrm{i} 1}+\mathrm{c}_{\mathrm{i} 2}\right)+\ldots+\mathrm{p}_{\mathrm{in}}\left(\mathrm{c}_{\mathrm{i} 1}+\mathrm{c}_{\mathrm{i} 2}+\ldots+\mathrm{c}_{\mathrm{in}}\right)
$$

ou pela expressão equivalente

$$
\mathrm{E}\left(\mathrm{C}_{\mathrm{T}}\right)=\mathrm{c}_{\mathrm{i} 1}+\mathrm{c}_{\mathrm{i} 2}\left(1-\mathrm{p}_{\mathrm{i} 1}\right)+\mathrm{c}_{\mathrm{i} 3}\left(1-\mathrm{p}_{\mathrm{i} 1}-\mathrm{p}_{\mathrm{i} 2}\right)+\ldots+\mathrm{c}_{\mathrm{in}}\left(1-\mathrm{p}_{\mathrm{i} 1}-\mathrm{p}_{\mathrm{i} 2}-\ldots-\mathrm{p}_{\mathrm{i}(\mathrm{n}-1)}\right)
$$

A fórmula (1) pode ser facilmente entendida reconhecendo que o comprimento da seqüência — ou o número $k$ de testes realizados — é um evento aleatório, cuja probabilidade é a probabilidade do $i_{k}$-ésimo teste ter resultado positivo; o custo correspondente a este 
comprimento é dado pela soma do custos dos $k$ testes realizados. A fórmula (2) calcula o custo em etapas: a cada etapa (final de um teste), dois eventos são possíveis: interrupção do processo, ou realização do próximo teste (e correspondente incremento no custo total).

Estas expressões fornecem o custo esperado antes de iniciar os testes. Ao longo do tempo, o valor esperado do custo se altera cada vez que um teste é realizado, mas é fácil mostrar que, se o teste tem resultado negativo, a ordem de execução dos testes restantes que minimiza o custo total permanece invariável. Assim, embora o ponto de interrupção da seqüência permaneça aleatório, a seqüência ótima pode ser completamente determinada a priori.

\subsection{Solução}

Demonstra-se que a seqüência de execução dos testes que minimiza o custo esperado $\mathrm{E}\left(\mathrm{C}_{\mathrm{T}}\right)$ é aquela em que os testes estão ordenados por ordem crescente das razões entre seus custos e as probabilidades de terem resultado positivo; em outras palavras, é a seqüência $\left(t_{i 1}, t_{i 2}, \ldots t_{i n}\right)$ tal que, para todo $\mathrm{k} \in\{1,2, \ldots, \mathrm{n}-1\}$ :

$$
\mathrm{c}_{\mathrm{ik}} / \mathrm{p}_{\mathrm{ik}} \leq \mathrm{c}_{\mathrm{i}(\mathrm{k}+1)} / \mathrm{p}_{\mathrm{i}(\mathrm{k}+1)}
$$

Para não sobrecarregar a exposição, a demonstração encontra-se no Apêndice.

Dada a extrema simplicidade desta solução, os autores pesquisaram a literatura, em busca de referência prévia ao problema e à própria solução; não se pretende afirmar que esta não exista, mas nada se conseguiu localizar.

O Quadro 1 sintetiza a definição formal do problema e a solução.

É interessante observar que, se os custos dos testes forem iguais, esta solução se reduz a uma das "heurísticas" (entre aspas, pois no caso, não é uma heurística, mas sim a definição da solução ótima) adotadas por Goulart et al. (1994).

Quadro 1 - Seqüência de custo esperado mínimo

- conjunto de diagnósticos possíveis $\left\{\mathrm{d}_{1}, \mathrm{~d}_{2}, \ldots \mathrm{d}_{\mathrm{n}}\right\}$

(mutuamente exclusivos e exaustivos)

- cada diagnóstico com probabilidade a priori $\mathbf{p}_{\mathbf{i}}$

- para cada diagnóstico $\mathbf{d}_{\mathbf{i}}$ existe apenas um teste $\mathbf{t}_{\mathbf{i}}$, com custo $\mathbf{c}_{\mathbf{i}}$ (teste positivo: diagnóstico $=\mathrm{d}_{\mathrm{i}}$ com $100 \%$ de certeza;

teste negativo: com $100 \%$ de certeza o diagnóstico não é $\mathrm{d}_{\mathrm{i}}$ )

- testes interrompidos ao primeiro resultado positivo

$=>$ seqüência de custo esperado mínimo: $\left(\mathrm{t}_{\mathrm{i} 1}, \mathrm{t}_{\mathrm{i} 2}, \ldots \mathrm{t}_{\mathrm{in}}\right)$ tal que:

para todo $\mathrm{k} \in\{1,2, \ldots, \mathrm{n}-1\}$ :

$$
\mathrm{c}_{\mathrm{ik}} / \mathrm{p}_{\mathrm{ik}} \leq \mathrm{c}_{\mathrm{i}(\mathrm{k}+1)} / \mathrm{p}_{\mathrm{i}(\mathrm{k}+1)}
$$




\subsection{Extensão para um problema com testes em diversos níveis}

O modelo e solução acima podem facilmente ser estendidos ao caso em que as causas ou sintomas se agrupam por grandes categorias, por estágio do processo, ou segundo algum outro critério, levando a uma classificação hierárquica em dois ou mais níveis. (Para exemplos de problemas deste tipo, ver Banks (1989), seção 14.5). Suponha então que, além dos testes que determinam diagnósticos individuais, há testes que reduzem os diagnósticos a um subconjunto (ou identificam um grupo de causas que contém a causa responsável pela falha ou problema). Suponha ainda que os subconjuntos, ou grupos, são mutuamente exclusivos (disjuntos). No caso de dois níveis, a diagnose ocorre em duas etapas: primeiro, realizam-se os testes do nível mais alto, para identificar o grupo de diagnósticos que contém o diagnóstico verdadeiro; em seguida, realizam-se os testes que irão determinar o diagnóstico final dentro do grupo identificado. A seqüência ótima dos testes em cada etapa pode ser determinada pelo procedimento acima proposto. Analogamente, se a hierarquia de causas (diagnósticos) tiver mais de dois níveis, o problema pode ser decomposto e solucionado na forma proposta, para um nível de cada vez, indo do mais alto para o mais baixo. Pode-se demonstrar formalmente que este método leva à solução de custo mínimo para o problema global; por considerarmos este resultado intuitivo, sua demonstração é aqui omitida, mas ela pode ser encontrada em Pires (1995).

\section{Caso em que os Diagnósticos Não São Mutuamente Exclusivos}

Foi visto que uma das hipóteses básicas do modelo desenvolvido é que os diagnósticos sejam mutuamente exclusivos. Será apresentada agora uma extensão do modelo para o caso em que essa condição não se verifica. As demais hipóteses, porém, continuam sendo mantidas. Especificamente, continua-se exigindo que: a união dos diagnósticos corresponda ao espaço amostral; os testes não possuam probabilidade de erro; o resultado positivo de qualquer teste, isoladamente, seja suficiente para identificar um diagnóstico; e a seqüência de testes seja interrompida ao primeiro resultado positivo.

Um exemplo de problema real apresentando essas condições será descrito mais adiante, na Seção 4. Foi precisamente tal problema a motivação para o desenvolvimento dessa extensão.

\subsection{Extensão do modelo matemático}

O fato de os diagnósticos não serem mutuamente exclusivos pode ser representado por uma mudança na definição de $\mathrm{p}_{\mathrm{j}}$ na fórmula do custo esperado. A nova definição passa a ser:

$\mathrm{p}_{\mathrm{j}}$ : probabilidade do teste $\mathrm{t}_{\mathrm{j}}$ ser positivo condicionada a os testes anteriores terem sido negativos, multiplicada pela probabilidade de os testes anteriores serem negativos. Em outras palavras, $p_{j}$ é a probabilidade da diferença de eventos $A-B$, onde $A=d_{j}$ e $B=$ união dos diagnósticos associados aos testes que precedem $t_{j}$ na seqüência (i.e., união de todos os $d_{i}$ tais que $t_{i}$ precede $t_{j}$ ).

Note que esta é a definição genérica para $\mathrm{p}_{\mathrm{j}}$, que, no caso particular em que os diagnósticos são mutuamente exclusivos, se reduz à probabilidade absoluta do diagnóstico (definição anterior para $\mathrm{p}_{\mathrm{j}}$ ).

O impacto desta nova definição de $\mathrm{p}_{\mathrm{j}}$ no método de solução é que as probabilidades $\left\{\mathrm{p}_{\mathrm{ik}}\right\}$ nas fórmulas (1) e (2) não correspondem mais apenas a permutações de um conjunto fixo de 
valores $\left\{\mathrm{p}_{\mathrm{j}}\right\}$, mas os próprios valores dos elementos deste conjunto $\left\{\mathrm{p}_{\mathrm{j}}\right\}$ variam agora de seqüência para seqüência.

Isto dificulta a determinação da seqüência ótima, pois as razões $c_{j} / p_{j}$ passam também a não mais possuir um valor constante para cada teste $t_{\mathrm{j}}$, mas a depender da seqüência.

Contudo, para qualquer teste $t_{j}$ é verdade que, em qualquer seqüência,

$$
\mathrm{p}_{\mathrm{j} \min } \leq \mathrm{p}_{\mathrm{j}} \leq \mathrm{p}_{\mathrm{j} \max }
$$

onde

$\mathrm{p}_{\mathrm{j} m i n}$ é a probabilidade de $\mathrm{t}_{\mathrm{j}}$ ter resultado positivo isoladamente (i.e., probabilidade de o diagnóstico ser $\mathrm{d}_{\mathrm{j}}$ e apenas $\mathrm{d}_{\mathrm{j}}$ );

$\mathrm{p}_{\mathrm{j} \max }$ é a probabilidade total de $\mathrm{t}_{\mathrm{j}}$ ter resultado positivo (independentemente do resultado dos demais testes; i.e., probabilidade total do diagnóstico $\mathrm{d}_{\mathrm{j}}$ ).

Então, por exemplo, em uma seqüência em que $t_{j}$ seja o último teste $\left(i_{n}=j\right), p_{j}=p_{j m i n}$; e numa seqüência em que $t_{j}$ seja o primeiro teste $\left(i_{1}=j\right), p_{j}=p_{j \max }$.

Estas condições são suficientes, mas não necessárias. I.e.,

$$
\begin{aligned}
& \mathrm{i}_{\mathrm{n}}=\mathrm{j} \Rightarrow \mathrm{p}_{\mathrm{j}}=\mathrm{p}_{\mathrm{j} \text { min }} \\
& \mathrm{i}_{1}=\mathrm{j} \Rightarrow>\mathrm{p}_{\mathrm{j}}=\mathrm{p}_{\mathrm{j} \text { max }}
\end{aligned}
$$

Em outras situações ( $\mathrm{i}_{\mathrm{k}}=\mathrm{j}, 1<\mathrm{k}<\mathrm{n}$ ), vale a condição em (4).

De (4), tira-se que, em qualquer seqüência, para cada $\mathrm{c}_{\mathrm{ik}} / \mathrm{p}_{\mathrm{ik}}, \mathrm{i}_{\mathrm{k}} \in\{1,2, \ldots, \mathrm{n}\}$ :

$$
\mathrm{c}_{\mathrm{ik}} / \mathrm{p}_{\mathrm{ik} \max } \leq \mathrm{c}_{\mathrm{ik}} / \mathrm{p}_{\mathrm{ik}} \leq \mathrm{c}_{\mathrm{ik}} / \mathrm{p}_{\mathrm{ik} \min }
$$

Se a ordenação dos $\mathrm{c}_{\mathrm{ik}} / \mathrm{p}_{\mathrm{ik}}$ pelo critério $\mathrm{c}_{\mathrm{ik}} / \mathrm{p}_{\mathrm{ik}}<\mathrm{c}_{\mathrm{i}(\mathrm{k}+1)} / \mathrm{p}_{\mathrm{i}(\mathrm{k}+1)}$ para todo $\mathrm{i}_{\mathrm{k}}$ for insensível à variação de cada $c_{j} / p_{j}$ entre os seus limites (i.e., se houver uma única seqüência em que $\mathrm{c}_{\mathrm{ik}} / \mathrm{p}_{\mathrm{i} k \min }<\mathrm{c}_{\mathrm{i}(\mathrm{k}+1)} / \mathrm{p}_{\mathrm{i}(\mathrm{k}+1) \max }$ para todo $k$ ), então esta é a seqüência ótima.

Se um subconjunto $S_{1}$ de testes tiver todos $c_{j} / p_{j m i n}$ inferiores a todos $c_{j} / p_{j \max }$ dos testes de um outro subconjunto $S_{2}$, então sabe-se que todos os testes de $S_{1}$ precedem todos os testes de $S_{2}$; e assim tem-se a priori uma ordem parcial dos testes na seqüência ótima.

Desta forma, o exame dos limitantes superior e inferior para as razões $c_{j} / p_{j}$ (iguais, respectivamente, a $\mathrm{c}_{\mathrm{j}} / \mathrm{p}_{\mathrm{j} \min }$ e $\mathrm{c}_{\mathrm{j}} / \mathrm{p}_{\mathrm{j} \max }$ ) pode vir a reduzir o espaço de busca da seqüência ótima, determinando grupos de testes que precedem outros grupos.

Uma vez assim definida uma ordem parcial dos testes, para determinar a sequêencia ótima resta apenas determinar a ordem ótima dos testes internos a cada grupo. Para isso, em princípio, o único procedimento geral é calcular e comparar os custos esperados das seqüências produzidas por todas as permutações possíveis destes testes. De qualquer forma, a subdivisão prévia do conjunto $\left\{\mathrm{t}_{\mathrm{j}}\right\}$ em grupos já proporciona uma redução evidente do número de seqüências alternativas em relação ao conjunto formado por todas as permutações de todos os testes do conjunto. Há, porém, um recurso adicional que pode reduzir ainda mais o número de alternativas a considerar dentro de um grupo de testes: os limitantes superior e inferior para as razões $c_{j} / p_{j}$ correspondentes aos testes do grupo podem ser recalculados, pois já se sabe que, qualquer que seja a seqüência, o conjunto dos testes que precederão cada teste do grupo será formado por: no mínimo (caso o teste seja o primeiro do grupo), todos os testes dos demais grupos precedentes; no máximo (caso o teste seja o último do grupo), todos 
estes, e mais todos os demais testes do próprio grupo. Assim, o exame destes novos valores para os limitantes das razões $c_{j} / p_{j}$ dentro do grupo pode definir uma ordem parcial dentro do grupo, particionando-o por sua vez em subgrupos, ou mesmo, por vezes, pode definir uma ordem total, determinando completamente a seqüência ótima dentro do grupo.

\subsection{Algoritmo}

Estas observações já são suficientes para definir um algoritmo geral para solução do problema. Este é apresentado no Quadro 2, abaixo.

Quadro 2 - Algoritmo para o caso de testes não mutuamente exclusivos

Dados:

- conjunto de diagnósticos possíveis $\left\{\mathrm{d}_{1}, \mathrm{~d}_{2}, \ldots \mathrm{d}_{\mathrm{n}}\right\}$

- probabilidade de cada diagnóstico e de cada interseção de diagnósticos (que podem ser estimadas pelas freqüências relativas dos eventos respectivos)

- custo de cada teste $\mathrm{t}_{\mathrm{j}}$ : formando o conjunto $\left\{\mathrm{c}_{1}, \mathrm{c}_{2}, \ldots \mathrm{c}_{\mathrm{n}}\right\}$

Passo 1: Para todo $j$, determinar $\mathrm{p}_{\mathrm{j} \min }$ e $\mathrm{p}_{\mathrm{j} m a x}$ :

$\mathrm{p}_{\mathrm{j} \text { min }}=$ probabilidade (ou freqüência relativa) de ocorrência do resultado positivo do teste correspondente conjuntamente com (interseção) resultado negativo de todos os demais;

$\mathrm{p}_{\mathrm{j} \text { max }}=$ probabilidade absoluta (ou freqüência relativa total) de ocorrência do resultado positivo do teste correspondente (sem considerar quais sejam os resultados dos demais testes);

Passo 2: Para todo $j$, calcular as razões $\mathrm{c}_{\mathrm{j}} / \mathrm{p}_{\mathrm{j} \min }$ e $\mathrm{c}_{\mathrm{j}} / \mathrm{p}_{\mathrm{j} \max }$;

Passo 3: Ordenar as razões $\mathrm{c}_{\mathrm{j}} / \mathrm{p}_{\mathrm{j} \text { min }}$; ordenar as razões $\mathrm{c}_{\mathrm{j}} / \mathrm{p}_{\mathrm{j} \text { max }}$; formam-se assim 2 seqüências;

Passo 4: Inspecionar a seqüência dos $c_{\mathrm{j}} / \mathrm{p}_{\mathrm{j} \text { min }}$ e a seqüência dos $\mathrm{c}_{\mathrm{j}} / \mathrm{p}_{\mathrm{j} \text { max }}$; determinar o segmento inicial de menor comprimento possível de cada uma das duas seqüências, que satisfaça as seguintes condições: o segmento de uma e de outra seqüência devem ter igual comprimento, e devem conter exatamente o mesmo conjunto de índices $j$ (embora não na mesma ordem); este conjunto de índices (que pode ser unitário) define o primeiro conjunto de testes na ordem parcial dos testes;

Passo 5: Repetir o Passo 4 para os testes restantes, definindo o segundo conjunto de testes na ordem parcial dos testes; e assim sucessivamente, até não restarem mais testes; está determinada a ordem parcial dos testes;

Passo 6: Caso todos os conjuntos sejam unitários, está determinada a seqüência ótima, e o algoritmo termina; caso contrário, ir para o Passo 7;

Passo 7: Para cada conjunto não unitário, determinar a seqüência dos testes internos ao conjunto. Isso pode ser feito segundo 2 procedimentos: 


\section{Procedimento A:}

- enumerar todas as seqüências possíveis de testes internos ao conjunto (permutações dos índices do conjunto);

- para cada seqüência assim definida (de testes do conjunto), concatená-la à seqüência já determinada dos testes que precedem o conjunto, e calcular a contribuição para $\mathrm{E}\left(\mathrm{C}_{\mathrm{T}}\right)$ devida à seqüência assim formada; (pode-se calcular apenas a contribuição dos testes do conjunto em análise para o custo esperado: sendo $r$ o número de testes do conjunto, e $m$, o número de testes que precedem o conjunto, trata-se da $(m+1)$-ésima até a $(m+r)$-ésima parcelas da fórmula (1) ou (2));

- comparar as contribuições para o custo esperado de cada seqüência assim definida (de testes do conjunto), e selecionar a seqüência de menor contribuição; com isto o conjunto está totalmente ordenado;

\section{Procedimento B:}

- para cada teste do conjunto, recalcular os limitantes $\mathrm{p}_{\mathrm{j} \min } \mathrm{e} \mathrm{p}_{\mathrm{j} \text { max }}$, da seguinte forma:

$\mathrm{p}_{\mathrm{j} \max }=\mathrm{P}\left(\mathrm{d}_{\mathrm{j}}-\cup\left\{\right.\right.$ todos os $\mathrm{d}_{\mathrm{j}}$ que precedem o conjunto $\left.\}\right)$;

$\mathrm{p}_{\mathrm{j} \min }=\mathrm{P}\left(\mathrm{d}_{\mathrm{j}}-\cup\left\{\right.\right.$ todos os $\mathrm{d}_{\mathrm{j}}$ que precedem o conjunto $\} \cup\left\{\right.$ todos os demais $\mathrm{d}_{\mathrm{j}}$ do próprio conjunto $\})$;

- para cada teste do conjunto, recalcular as razões $\mathrm{c}_{\mathrm{j}} / \mathrm{p}_{\mathrm{j} \min }$ e $\mathrm{c}_{\mathrm{j}} / \mathrm{p}_{\mathrm{j} \max }$;

- proceder, dentro do conjunto, como nos passos 3, 4, e 5. Três situações são possíveis:

- chegou-se a uma seqüência única: neste caso, o conjunto está ordenado;

- não se conseguiu subdividir o conjunto: neste caso, é preciso aplicar o procedimento A; após o qual, o conjunto estará ordenado;

- o conjunto foi dividido em subconjuntos menores: para o $1^{\mathrm{o}}$ subconjunto, utilizar o procedimento A; para os demais, pode-se: ou utilizar o procedimento A diretamente, ou reaplicar o procedimento B para subdividir o subconjunto, o que equivale a reaplicar o Passo 7 recursivamente; observe-se, porém, que a eficiência do procedimento B deve começar a diminuir: são cada vez menores as chances de conseguir subdividir o subconjunto, e, mesmo quando o subconjunto for subdividido, será cada vez menor o ganho em termos de redução do número de alternativas. Para cada subconjunto que não se conseguir subdividir, aplicar o procedimento $\mathrm{A}$, que ordena totalmente o subconjunto;

quando o conjunto estiver totalmente ordenado, passar para o conjunto seguinte e voltar ao Passo 7;

Passo 8 (critério de parada): Quando todos os conjuntos estiverem, internamente, totalmente ordenados, a sua concatenação define a seqüência ótima. 


\section{Uma Aplicação a um Caso Real}

\subsection{Descrição do caso}

Passemos agora a uma breve descrição do caso que motivou extensão do método. Tratou-se da determinação da seqüência ótima de testes sorológicos no banco de sangue (BS) de um hospital universitário. (Para uma descrição mais detalhada, ver Santos Jr., 1997).

O sangue de cada doador só pode ser utilizado após passar pela triagem sorológica, composta de 8 diferentes testes laboratoriais para identificar a presença de doenças infecto-contagiosas. Os resultados destes testes são considerados $100 \%$ confiáveis. Os custos com estes testes representam $71,22 \%$ dos custos incorridos pelo BS.

Ao terem os autores contato com o BS, o procedimento adotado era realizar todos os 8 testes, em paralelo. Contudo, lembrando que basta que um dos exames tenha resultado positivo para rejeitar o sangue do doador, surgiu a idéia, para tentar reduzir os custos, de aplicar os testes em seqüência, interrompendo-os após o primeiro diagnóstico positivo.

O problema do BS, portanto, parecia ser bem descrito pelo modelo matemático da Seção 2.1, representado no Quadro 1; a proposta de solução, então, foi determinar a seqüência de custo esperado mínimo, e passar a realizar os testes nessa seqüência.

Apenas, note-se que, no caso do BS, a união dos 8 diagnósticos não exaure o espaço amostral: há o evento "apto" (sangue em condições de ser utilizado), que constitui o complemento desta união. Como para os aptos são feitos todos os testes (não se pode utilizar sangue que não tenha "passado" em todos os testes), o seqüenciamento só terá efeito de reduzir os custos dos exames dos candidatos não aptos; para os aptos, o custo é fixo e máximo. (Para os aptos, a fórmula (1) não vale, e a fórmula (2) vale, $\operatorname{com} p_{i}=0$ para todo $i, \mathrm{o}$ que reduz (2) trivialmente a $\mathrm{E}\left(\mathrm{C}_{\mathrm{T}}\right)=\Sigma \mathrm{c}_{\mathrm{i}}$ ). Trabalhou-se, assim, com as probabilidades de ocorrência das doenças consideradas condicionadas à presença de pelo menos uma das delas.

Como as despesas com o pessoal do laboratório são fixas, independentemente do seu grau de utilização ou tempo ocioso, para efeitos da determinação da seqüência de custo esperado mínimo, considerou-se como custo de cada exame apenas o custo do material ("kit" para o teste). Para estimar as probabilidades $\mathrm{p}_{\mathrm{j}}$, levantaram-se e (a partir dos dados históricos disponíveis) as freqüências absolutas de ocorrência dos diversos diagnósticos. Estes custos e freqüências absolutas constam, respectivamente, das Tabelas 1 e 2.

Tabela 1 - Custos dos testes $(\mathrm{R} \$)$

\begin{tabular}{|c|c|c|c|c|c|c|c|c|}
\hline teste & $\mathbf{3}$ & $\mathbf{1}$ & $\mathbf{5}$ & $\mathbf{2}$ & $\mathbf{4}$ & $\mathbf{6}$ & $\mathbf{8}$ & $\mathbf{7}$ \\
\hline custo (R\$) & 3,33 & 0,14 & 11,44 & 2,19 & 0,07 & 5,83 & 4,67 & 3,25 \\
\hline
\end{tabular}

Tabela 2 - Freqüências de ocorrência dos diagnósticos

\begin{tabular}{|c|c|c|c|c|c|c|c|c|c|c|c|c|c|}
\hline diagnóstico & $\mathbf{3}$ & $\mathbf{1}$ & $\mathbf{5}$ & $\mathbf{1 + 3}$ & $\mathbf{2 + 3}$ & $\mathbf{7}$ & $\mathbf{8}$ & $\mathbf{3 + 4}$ & $\mathbf{3}+\mathbf{5}$ & $\mathbf{4}$ & $\mathbf{6}$ & $\mathbf{2}$ & $\mathbf{3 + 6}$ \\
\hline freq. absoluta & 276 & 24 & 12 & 11 & 08 & 04 & 04 & 04 & 03 & 03 & 02 & 02 & 02 \\
\hline
\end{tabular}

(Número total de candidatos examinados: 355 ) 
Observando a Tabela 2, nota-se que uma das hipóteses básicas do modelo não se verifica: os diagnósticos, no caso, não são mutuamente exclusivos. A Figura 1 apresenta um diagrama de Venn dos resultados positivos para os testes. O fundo da figura corresponde ao evento "apto".

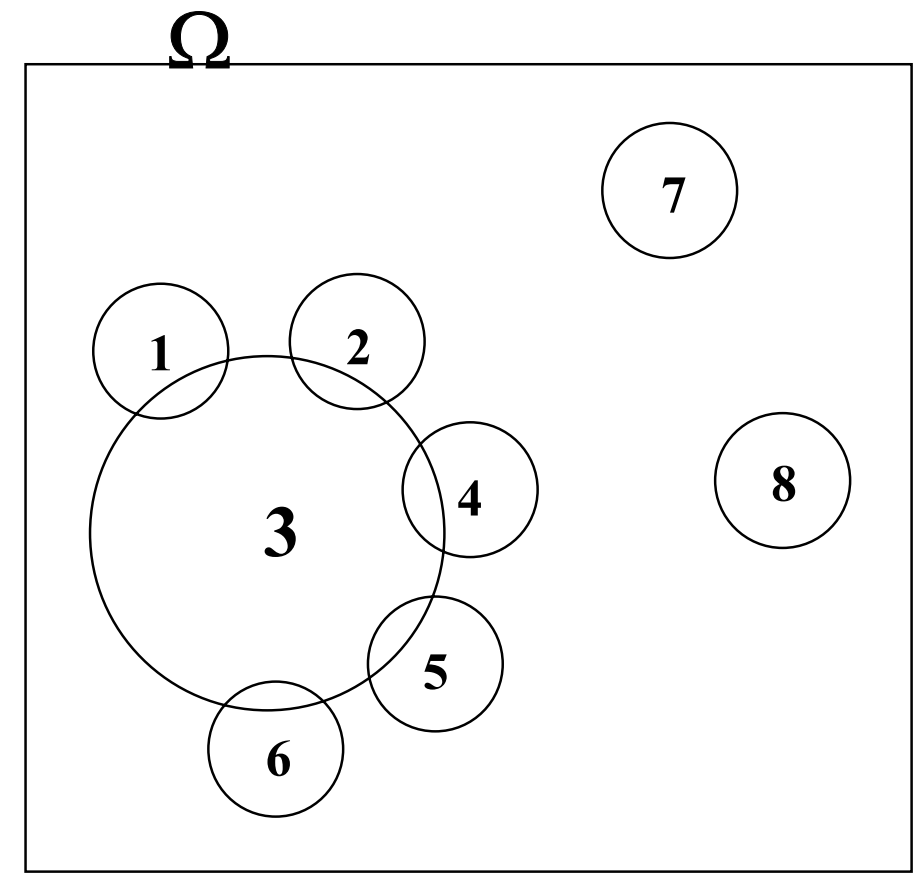

Figura 1 - Diagrama de Venn dos eventos "resultado positivo" nos diversos exames

Dada a violação desta hipótese básica, para poder solucionar o problema, foi necessária a extensão do modelo e do método de solução descrita na Seção 3.

\subsection{Solução}

O algoritmo no Quadro 2 foi aplicado ao caso do BS, determinando a seguinte seqüência ótima de realização dos testes laboratoriais:

$$
S_{\text {ótima }}=\left(t_{1}, t_{4}, t_{3}, t_{7}, t_{5}, t_{2}, t_{8}, t_{6}\right)
$$

A aplicação detalhada, passo a passo, do algoritmo, pode ser encontrada em Epprecht et al. (1999).

O custo esperado desta seqüência (que é o custo esperado ótimo) é igual a 4,1645 (R\$), o que representa apenas $13,47 \%$ do custo do conjunto completo de testes (que é de $\mathrm{R} \$ 30,92$ ). Assim, a utilização dos testes em seqüência, aguardando o resultado de um teste antes de decidir realizar o seguinte, proporciona uma economia esperada de $86,53 \%$ no custo dos exames dos candidatos sem condições de doar.

Como a proporção de candidatos sem condições de doar é de 17,53\%, e lembrando que $71,22 \%$ dos custos incorridos pelo BS são com os exames, esta economia corresponde a $0,8653 \times 0,1753 \times 71,22 \%=10,8 \%$ de todos os custos do BS. 


\subsection{Questões de ordem prática para implementação dos resultados}

O seqüenciamento dos testes tem um impacto sobre os procedimentos, inclusive provocando um aumento da duração da triagem sorológica (o tempo total dos testes, que, com os testes em paralelo, é inferior a 4 horas, aumentará, com os testes em seqüência, para cerca de 13 horas). Isto não necessariamente levará a atrasos significativos na produção, pois o processo de coleta e exames só é realizado uma vez por dia, mas pode impactar nos turnos de trabalho; implicar, por exemplo, em que os testes sejam iniciados por uma equipe e terminados por outra. Estas questões devem ser analisadas pelo BS, em termos de custo e benefício. Mas algumas alternativas, que podem representar um bom compromisso entre o ganho teórico e a viabilidade prática, são fornecidas com a análise de sensibilidade a seguir.

\subsection{Análise de sensibilidade}

Devido aos possíveis problemas de implementação da solução ótima, é interessante realizar uma análise de sensibilidade dos resultados, para calcular a redução de custos que se obteria com um procedimento alternativo, sub-ótimo em termos de custo esperado, mas que requereria um tempo total para a execução dos testes bem inferior a 13 horas; a saber: realizar os primeiros testes da seqüência ótima efetivamente em seqüência, e em seguida realizar os demais (que raramente terão resultado positivo) em paralelo.

Para esta análise basta calcular o custo esperado das "seqüências" assim determinadas, de maneira análoga ao que foi feito para a seqüência ótima. Os resultados são:

- Testes 1, 4 e 3 nesta ordem, seguidos dos demais em paralelo: $\mathrm{E}\left(\mathrm{C}_{\mathrm{T}}\right)=\mathrm{R} \$ 4,985$

( $20 \%$ mais cara que a seqüência ótima; $84 \%$ de economia em relação ao procedimento vigente de efetuar todos os testes em paralelo)

- Teste 1, depois teste 3, seguidos dos demais em paralelo:

$\mathrm{E}\left(\mathrm{C}_{\mathrm{T}}\right)=\mathrm{R} \$ 5,229$

( $25 \%$ mais cara que a seqüência ótima; $83 \%$ de economia em relação ao procedimento vigente de efetuar todos os testes em paralelo)

Portanto, um procedimento sub-ótimo destes pode levar a economias bem próximas à proporcionada pela seqüência ótima, com um aumento bem menor da duração da triagem sorológica; e isto pode fazer a diferença entre uma solução ótima mas inviável e uma solução quase ótima viável.

Sobre a precisão das estimativas das probabilidades envolvidas, Epprecht et al. (1999) apresentam uma discussão (aqui omitida por razões de espaço), concluindo que os resultados são bastante robustos.

\section{Conclusões e Comentários Finais}

\subsection{Resultados da aplicação ao problema real}

A aplicação do método ao problema do banco de sangue mostrou a possibilidade de uma economia de cerca de $86 \%$; i.e., a utilização da seqüência ótima obtida para os testes constitui um procedimento 7 vezes mais barato que o procedimento vigente. Considerando possíveis dificuldades de ordem prática para implementação da solução, foram sugeridas 
duas alternativas (mais viáveis) que levam a economias menores, mas ainda substanciais: da ordem de 83 ou $84 \%$ (i.e., procedimento cerca de 6 vezes mais barato que o vigente).

\subsection{Eficiência do algoritmo}

A aplicação do algoritmo proporcionou uma enorme redução do número de alternativas a considerar: no lugar das 40320 seqüências possíveis de 8 testes, foi necessário calcular o custo esperado de apenas 2 subseqüências de 3 testes (para detalhes, ver Epprecht et al., 1999). Embora este caso exemplifique o ganho proporcionado pelo uso do algoritmo (em relação à busca exaustiva no espaço de soluções), não se pretende generalizar este resultado: o grau de redução do número de seqüências alternativas a considerar dependerá, para cada problema específico, das probabilidades dos diagnósticos e de suas interseções. $\mathrm{O}$ caso analisado foi extremamente favorável ao desempenho do algoritmo, já que apenas um teste $\left(\mathrm{t}_{3}\right)$ possuía interseções com vários outros. De qualquer forma, é razoável esperar reduções substanciais do número de alternativas cada vez que se subdividir o conjunto $\left\{\mathrm{t}_{\mathrm{j}}\right\}$ em subconjuntos, ordenados uns em relação aos outros.

\subsection{Aplicabilidade do modelo e algoritmo}

Como foi dito, o modelo não se restringe a diagnósticos médicos, mas é genérico. Sempre que o problema puder ser descrito pelas condições no Quadro 1, a solução indicada no mesmo Quadro 1 se aplica. Sempre que a única violação daquelas condições for a não exclusividade dos diagnósticos, mas o procedimento seja interromper a seqüência ao obter um diagnóstico, pode-se aplicar o algoritmo no Quadro 2.

Outra situação, ligeiramente mais complexa, que pode ser tratada pelo modelo no Quadro 1, é a de testes organizados em níveis hierárquicos: neste caso, o procedimento de solução deve ser aplicado separadamente para cada nível de testes. Este caso é mencionado em Epprecht \& Pires (1995); uma discussão mais detalhada, com um argumento que mostra que o problema é perfeitamente decomponível em um problema independente para cada nível, pode ser encontrada em Pires (1995).

É importante insistir neste ponto: o modelo assume que os testes são interrompidos sempre que se chegar a um (primeiro) diagnóstico, inclusive no caso em que os diagnósticos não são mutuamente exclusivos. Como se vê, este é exatamente o caso do problema do banco de sangue aqui analisado. Para problemas em que este procedimento não seja obedecido, o modelo não se aplica.

No caso de diagnósticos de falhas de equipamentos (ou diagnósticos de problemas em processos, no contexto de controle de qualidade) em que a estratégia seja interromper os testes e reiniciar a operação do equipamento (ou processo) assim que uma (primeira) causa for identificada e eliminada, há uma nuance: o modelo se aplica apenas à minimização do custo esperado de identificar esta causa única ou primeira. Caso o problema persista, ou se evidencie a presença de outro problema, e seja necessário prosseguir com os testes, a simples retomada da seqüência calculada pelo modelo a partir do ponto em que foi interrompida pode não mais representar a solução ótima, pois a probabilidade de resultado positivo para cada teste considerada nas fórmulas de $\mathrm{E}\left(\mathrm{C}_{\mathrm{T}}\right)$ é condicionada a todos os testes anteriores terem tido resultado negativo. Mesmo assim, se as probabilidades de interseções de diagnósticos forem muito pequenas em relação às probabilidades absolutas dos diagnósticos, os resultados não deverão ser muito sensíveis a esta inadequação do modelo, e pode-se esperar que a solução (seqüência) indicada pelo modelo, se não for a solução ótima, seja uma boa solução. 
Outra maneira de enxergar esta questão é que, se as probabilidades de interseções de diagnósticos forem muito pequenas em relação às probabilidades absolutas dos diagnósticos, então a probabilidade de ser necessário retomar a seqüência de testes após o primeiro diagnóstico positivo também será muito pequena. Assim, com grande probabilidade, as condições de aplicação do modelo são respeitadas e a solução é ótima; nas raras ocasiões em que isto não ocorrer, o modelo fornece uma solução ótima para a identificação da primeira causa, e uma boa solução para o prosseguimento da seqüência de testes.

Caso não se disponha de dados históricos que permitam estimar com razoável precisão as probabilidades de cada diagnóstico (e interseções de diagnósticos), podem-se utilizar estimativas subjetivas. Caso não se sinta confiança suficiente nestas estimativas para aplicação do algoritmo, e por isso, ou por quaisquer outras razões operacionais não representadas no modelo, a decisão sobre a seqüência de testes deva ser subjetiva, ainda assim o exame das razões $c_{j} / p_{j}$ (ou de estimativas subjetivas, ou de faixas de valores para elas) constitui um critério para auxílio à decisão, distinguindo pelo menos aqueles testes em que os valores destas razões sejam muito diferentes, e aprimorando assim o sentimento do decisor quanto à sensibilidade do custo total esperado à ordem dos testes.

Finalmente, ao usar como critério o valor esperado do custo, implicitamente o modelo não se destina a situações em que se incorrerá nos custos uma única vez ou raramente, tais como decisões individuais quanto a exames às próprias custas de um paciente, ou problemas raros de um equipamento. Para tais situações, pode ser indicado considerar, além do custo esperado, ou mesmo em vez dele, a dispersão da distribuição de probabilidades do custo total, a probabilidade de ocorrer um custo total excessivamente alto, ou o uso de funções de preferência ou utilidade do cliente. Contudo, para problemas de diagnóstico que se repitam com freqüência, como no caso aqui descrito do banco de sangue ou no caso de processos monitorados permanentemente, o custo esperado é o critério mais adequado, e suficiente para a decisão. Esta questão é bem conhecida, mas pode-se citar Gorentsin et al. (1992): "The use of the mean costs as the decision criterion in stochastic problems is adequate for 'high frequency' phenomena, in which a representative sample of all values is expected to occur (...)". Nestes casos, além de o critério ser adequado, é usualmente possível obter dados históricos suficientes para fornecer boas estimativas das probabilidades dos diagnósticos, para entrada no modelo.

\subsection{Direções de prosseguimento}

Dentre as extensões possíveis para o modelo, as mais naturais e relevantes vão na direção do relaxamento das hipóteses assumidas, de modo a admitir a possibilidade de diferentes causas (ou doenças) produzirem sintomas comuns, de modo que um teste não é suficiente para determinar um diagnóstico, mas um subconjunto de diagnósticos; ou ainda a admitir testes cujos resultados possuem uma margem (probabilidade) de erro; para citar apenas os aspectos mais evidentes.

É possível que algumas destas situações não admitam um algoritmo exato de solução, e requeiram (como nos trabalhos de Goulart et al., 1994, e Pereira, 1994) o uso de heurísticas ou outras técnicas (por exemplo técnicas de inteligência artificial), para estes casos, espera-se que o presente modelo possa ajudar a prover "insight" sobre a questão.

\section{Agradecimentos}

Este trabalho se insere em projetos de pesquisa dos dois primeiros autores, apoiados pelo CNPq, e na pesquisa de mestrado do terceiro autor, apoiado pela CAPES. 


\section{Referências Bibliográficas}

(1) Epprecht, E.K. \& Pires, D.M. (1995). Minimização de Custos em Diagnósticos de Falhas. XXVII Simpósio Brasileiro de Pesquisa Operacional, Vitória, ES.

(2) Epprecht, E.K.; Santos Jr.; M.C. \& Brasil, G.H. (1999). Um Modelo de Minimização de Custos em Diagnósticos com um Caso de Aplicação em um Banco de Sangue. Memorando Técnico $n^{\circ}$ 03/99, Departamento de Engenharia Industrial, PUC-Rio, Rio de Janeiro.

(3) Pires, D.M. (1995). Minimização de Custos em Diagnósticos de Falhas. Dissertação de Mestrado, PUC-Rio, Departamento de Engenharia Industrial, Rio de Janeiro.

(4) Banks, J. (1989). Principles of Quality Control. John Wiley \& Sons. New York.

(5) Gorentsin, B.G.; Campodonico, N.M.; Costa, J.P. \& Pereira, M.V.F. (1992). Power System Expansion Planning under Uncertainty. IEEE/Power Engineering Society 1992 Winter Meeting, New York, NY, Jan 1992.

(6) Goulart, Henrique E.; Fernandes, A.O. \& Luna, Henrique P.C. (1994). Ferramentas de Diagnóstico Automatizado. Anais do $10^{\circ}$ Congresso Brasileiro de Automática / $6^{\circ}$ Congresso Latino-Americano de Controle Automático, Rio de Janeiro, setembro de 1994.

(7) Pereira, Néocles A. (1994). Sistema Especialista de $2^{a}$ Geração para Diagnose Técnica: Modelo e Procedimento. Gestão e Produção, 1(1), abril 1994.

(8) Montgomery, Douglas C. (1997). Introduction to Statistical Quality Control. John Wiley \& Sons ( $3^{\mathrm{a}}$ ed.). New York.

(9) Santos Jr., M.C. dos (1997). Qualidade em Serviços: Estudo de Caso em um Banco de Sangue. Dissertação de Mestrado, PUC-Rio, Departamento de Engenharia Industrial, Rio de Janeiro.

\section{Apêndice: Demonstração do Teorema do Quadro 1}

Seja uma seqüência qualquer. Tomem-se 2 testes consecutivos nessa seqüência, $t_{\mathrm{k}}$ e $t_{\mathrm{k}+1}$, tais que a condição $c_{i k} / p_{i k} \leq c_{i(k+1)} / p_{i(k+l)}$ não se verifique, i.e., tais que $c_{i k} / p_{i k}>c_{i(k+1)} / p_{i(k+l)}$.

$\mathrm{O}$ custo esperado desta seqüência, notado $\mathrm{E}(\mathrm{C})_{\mathrm{ij}}$ (essa notação será esclarecida a seguir), pode ser escrito como:

$$
\mathrm{E}(\mathrm{C})_{\mathrm{ij}}=\sum_{x=1}^{k-1}\left(p_{i x} \sum_{y=1}^{x} c_{i y}\right)+p_{i k} \sum_{y+1}^{k} c_{i y}+p_{i(k+1)} \sum_{y=1}^{k+1} c_{i y}+\sum_{x=k+2}^{n}\left(p_{i x} \sum_{y=1}^{x} c_{i y}\right)
$$

Essa expressão é obtida da fórmula (1), simplesmente separando o somatório em 4 termos, de modo a isolar as parcelas que têm como coeficientes $p_{i k} \mathrm{e} p_{i(k+1)}$.

Expandindo a $2^{\mathrm{a}}$ e a $3^{\mathrm{a}}$ parcelas do membro direito, e chamando ainda $i_{\mathrm{k}}$ de $i$, e $i_{\mathrm{k}+1}$ de $j$, esta expressão se transforma em:

$$
\mathrm{E}(\mathrm{C})_{\mathrm{ij}}=\sum_{x=1}^{k-1}\left(p_{i x} \sum_{y=1}^{x} c_{i y}\right)+p_{i}\left(\sum_{y+1}^{k-1} c_{i y}+c_{i}\right)+p_{j}\left(\sum_{y+1}^{k-1} c_{i y}+c_{i}+c_{j}\right)+\sum_{x=k+2}^{n}\left(p_{i x} \sum_{y=1}^{x} c_{i y}\right)
$$


Agora explica-se a notação $\mathrm{E}(\mathrm{C})_{\mathrm{ij}}$ : eliminou-se o subscrito $\mathrm{T}$ do custo total $\mathrm{C}_{\mathrm{T}}$ apenas por simplicidade, e o índice $i j$ é usado para indicar que nesta seqüência o teste $t_{j}$ é o sucessor imediato do teste $t_{i}$.

Invertendo a ordem de execução dos testes $t_{i}$ e $t_{j}$ (i.e., permutando-os na seqüência), o custo esperado se tornará:

$$
\mathrm{E}(\mathrm{C})_{\mathrm{ji}}=\sum_{x=1}^{k-1}\left(p_{i x} \sum_{y=1}^{x} c_{i y}\right)+p_{j}\left(\sum_{y+1}^{k-1} c_{i y}+c_{j}\right)+p_{i}\left(\sum_{y+1}^{k-1} c_{i y}+c_{j}+c_{i}\right)+\sum_{x=k+2}^{n}\left(p_{i x} \sum_{y=1}^{x} c_{i y}\right)
$$

(O índice $j i$ no membro esquerdo indica que nesta seqüência o teste $t_{j}$ é executado imediatamente antes do teste $t_{i}$ ).

A diferença no custo esperado é, portanto, dada por:

$$
E(C)_{i j}-E(C)_{j i}=p_{j} c_{i}-p_{i} c_{j}
$$

Lembremos que, por hipótese, $c_{i} / p_{i}>c_{j} / p_{j}$. (Note ainda que todos os custos e probabilidades são positivos). Portanto, $p_{j} c_{i}>p_{i} c_{j}$. Assim, $E(C)_{i j}-E(C)_{j i}>0 \therefore$

$$
\therefore E(C)_{i j}>E(C)_{j i}
$$

Em outras palavras, permutando os testes $t_{i}$ e $t_{j}$, o custo esperado da seqüência diminui.

Como $i$ e $j$ são índices genéricos, sempre que dois testes consecutivos quaisquer $t_{i}$ e $t_{j}$ forem tais que $c_{i} / p_{i}>c_{j} / p_{j}$, a solução pode ser melhorada pela permutação destes testes. Só não será mais possível fazê-lo quando todos testes estiverem ordenados por ordem crescente dos respectivos $c_{k} / p_{k}$. Neste caso, qualquer permutação dos testes levaria a um aumento do custo esperado (pois voltaria a haver pelo menos um par de testes consecutivos tais que $\left.c_{i} / p_{i}>c_{j} / p_{j}\right)$, o que mostra que se chegou à solução de custo mínimo.

Note ainda que, a qualquer momento dentro da seqüência (i.e., após a execução de qualquer número de testes, a seqüência restante continua obedecendo à ordem crescente dos fatores $c_{i k} / p_{i k}$ : ela é de fato a seqüência que minimiza o valor esperado dos custos dos testes restantes. Assim, a seqüência ótima pode ser inteiramente determinada a priori. 